Doug Geisler, Eva K. Grebel, and Dante Minniti, eds.

\title{
Star Clusters and Stellar Populations of the M31 Disk as Viewed by HST
}

\author{
Benjamin F. Williams \\ University of Washington, Astronomy Box 351580, Seattle, WA 98195, \\ USA, ben@astro.washington.edu \\ Paul W. Hodge \\ University of Washington, Astronomy Box 351580, Seattle, WA 98195, \\ USA, hodge@astro.washington.edu
}

\begin{abstract}
We present a new method for finding associations of young stars in M31 using broadband WFPC2 data from the HST data archive. Applying our identification method to 13 WFPC2 fields, covering an area of $\sim 60$ arcmin $^{2}$, has revealed 80 new candidate young star clusters in these portions of the M31 disk. Most of these clusters are small ( $\$ 5 \mathrm{pc})$ young ( 10-200 Myr) star groups located within large OB associations. We have estimated the reddening values and the ages of each candidate individually by fitting isochrones to the stellar photometry. We provide a catalogue of the candidates including rough approximations of their reddenings and ages.
\end{abstract}

\section{Introduction}

Observations of extragalactic young star clusters are essential for understanding how star formation affects galaxy evolution. High-resolution photometric data and advances in computational analysis techniques have allowed objective identification of $\mathrm{OB}$ associations in galaxies, creating more uniform samples on which to perform statistical analyses. These uniform samples have been crucial to many recent advances in our understanding of the properties of extragalactic OB associations. For example, the similarity of their size distributions and stellar luminosity functions across different galaxy types (Bresolin et al. 1998), and their similar luminosity function across galaxy types (Battinelli et al. 2000) have suggested that massive star formation occurs by similar processes in all galaxies. At the same time, OB associations' average population sizes appear to differ between galaxies of different morphological type (Bresolin \& Kennicutt 1997), showing that environment has some effect on massive star formation.

M31 has been an excellent laboratory for the study of massive star formation due to its proximity and its many active spiral arms. These arms contain several hundred young open clusters and OB associations (Hodge 1979). These groups of young stars have been studied extensively from the ground and from space. Due to the crowding of stars and variable reddening in the disk, the optical ground based data was used mainly to map the structure of M31 OB associations. These 
studies have shown, for example, that massive star formation in M31 appears hierarchical (Battinelli et al. 1996). Space-based observations are allowing more detailed analyses of these regions of recent star formation. For example, Magnier et al. (1997) have used HST photometric data to determine the reddening distribution and ages of a handful of $\mathrm{OB}$ associations. In this paper, we expand upon their work with a new method for finding clusters within complex star forming regions.

\section{Identification and Analysis of Young Clusters of Stars}

We acquired and reduced 13 WFPC2 fields from the HST archive, each observed through at least the $\mathrm{B}$ and $\mathrm{V}$ broad-band filters. We determined instrumental magnitudes for each of these fields using the automated programs DAOPHOT II and ALLSTAR (Stetson, Davis, \& Crabtree 1990). We then obtained standard $\mathrm{U}, \mathrm{B}$, and $\mathrm{V}$ magnitudes from our instrumental magnitudes using the methods, zero points, and transformation coefficients given in Holtzman et al. (1995).

In order to explore the many open clusters and young stellar associations, we created an objective method for detecting the clusters within the fields. Using our stellar positions and photometry from DAOPHOT II and ALLSTAR, the mean surface density of bright $\left(m_{V}<24.5\right)$ blue $(B-V<0.45)$ stars is determined. Then the standard deviation from the mean density $(\sigma)$ on a size scale specified by the user is calculated. This calculation is performed by comparing the density of bright blue stars in regions of the specified size around every bright blue star detected in the field with the mean density. This calculation is followed by a search for regions containing at least 4 bright blue stars and having surface densities of bright blue stars $3 \sigma$ above the mean.

Finally, in order to remove statistical anomalies from our sample, we ran each cluster candidate through a surface brightness test. In this test, we measure the surface brightness around the center of each cluster candidate. This test was a bit more complex since, due to completeness issues, the centers of the stellar overdensities were not always aligned exactly with the high surface brightness regions. Therefore we allowed overdensities with very nearby high surface brightness regions to pass. This step required great care for the larger clusters since the chance of one bright star entering the surface brightness calculation and enhancing the surface brightness near the center of the overdensity was higher for the large clusters. We found the number of these single bright star contaminants was reduced when we removed candidates with substantial $\left(>2 \mathrm{mag} \operatorname{arcsec}^{-2}\right)$ increases in surface brightness away from the center. These large jumps in surface brightness were usually bright single point sources, which were likely foreground stars. Any stellar overdensity whose measured surface brightness characteristics did not pass our objective criteria was not likely part of an underlying cluster of unresolved stars. These low surface brightness regions were removed from the sample. 81 cluster candidates were found. The only previously cataloged blue star cluster in the survey region was G42 (Sargent et al. 1977), and it was found by our algorithm.

We estimated the ages and $E_{B-V}$ values for our cluster candidates by fitting the stellar evolution isochrones of Girardi et al. (2000) to the stars contained within the stellar overdensity. Since the average number of bright blue stars 
in areas the size that we were sampling was generally $\lesssim 1$, we assume that the stars we detected were cluster members. For the purposes of roughly estimating the cluster ages, we assumed these stars approximately represented the main sequence turnoff of the cluster. Errors for these estimates were determined experimentally using simulated data and modified jack-knife tests. Coordinates and estimated physical properties for a subsample of our cluster candidates are given in Table 1. For a full description of our methods and a complete list of cluster candidates found by this objective routine, see Williams \& Hodge (2001).

Table 1. Portion of our catalog of positions, age estimates, and reddening values for M31 young star cluster candidates. For the complete catalog of 81 candidates, see Williams \& Hodge (2001).

\begin{tabular}{ccccc}
\hline ID $^{a}$ & $\alpha(\mathrm{J} 2000)$ & $\delta(\mathrm{J} 2000)$ & $\log ($ age $)$ & $E_{B-V}$ \\
\hline M31SCC J003952+403141 & $0: 39: 52.4$ & $40: 31: 41.3$ & $8.00 \pm 0.2$ & $0.28 \pm 0.1$ \\
M31SCC J004000+403325 & $0: 40: 00.0$ & $40: 33: 25.0$ & $7.90 \pm 0.4$ & $0.22 \pm 0.1$ \\
M31SCC J004000+403406 & $0: 40: 00.8$ & $40: 34: 06.6$ & $7.75 \pm 0.45$ & $0.21 \pm 0.1$ \\
M31SCC J004001+403420 & $0: 40: 01.5$ & $40: 34: 20.1$ & $8.25 \pm 0.3$ & $0.20 \pm 0.2$ \\
M31SCC J004004+403440 & $0: 40: 04.7$ & $40: 34: 40.5$ & $8.10 \pm 0.2$ & $0.23 \pm 0.1$ \\
M31SCC J004006+403508 & $0: 40: 06.8$ & $40: 35: 08.4$ & $7.30 \pm 0.9$ & $0.25 \pm 0.1$ \\
M31SCC J004010+403624 & $0: 40: 10.4$ & $40: 36: 24.2$ & $7.75 \pm 0.6$ & $0.01 \pm 0.1$ \\
M31SCC J004012+403632 & $0: 40: 12.0$ & $40: 36: 32.6$ & $7.70 \pm 0.5$ & $0.24 \pm 0.1$ \\
M31SCC J004012+403617 & $0: 40: 13.0$ & $40: 36: 17.3$ & $7.90 \pm 0.4$ & $0.17 \pm 0.1$ \\
M31SCC J004013+403815 & $0: 40: 13.7$ & $40: 38: 15.5$ & $7.70 \pm 0.4$ & $0.43 \pm 0.1$ \\
M31SCC J004015+403652 & $0: 40: 15.4$ & $40: 36: 52.9$ & $7.90 \pm 0.2$ & $0.35 \pm 0.1$ \\
M31SCC J004032+403320 & $0: 40: 32.9$ & $40: 33: 20.5$ & $7.90 \pm 0.2$ & $0.16 \pm 0.1$ \\
M31SCC J004033+403308a & $0: 40: 33.3$ & $40: 33: 08.6$ & $7.75 \pm 0.2$ & $0.31 \pm 0.1$ \\
M31SCC J004033+403326 & $0: 40: 33.5$ & $40: 33: 26.9$ & $7.85 \pm 0.2$ & $0.30 \pm 0.1$
\end{tabular}

${ }^{a} \mathrm{M} 31 \mathrm{SCC}$ is an IAU registered acronym. M31SCC J004000+403406 is cluster G42 in the globular cluster catalogue of Sargent et al. (1977).

\section{References}

Battinelli, P., Efremov, Y., \& Magnier, E. A. 1996, A\&A, 314, 51

Battinelli, P. et al. 2000, A\&A, 357, 437

Bresolin, F. \& Kennicutt, R. C. 1997, ApJ, 113, 975

Bresolin, F., et al. 1998, AJ, 116, 119

Girardi, L., Bressan, A., Bertelli, G., \& Choisi, C. 2000, A\&AS141, 371

Hodge, P. W. 1979, AJ, 84, 744

Magnier, E. A. et al. 1997, MNRAS, 292, 490

Sargent, W. L. W., Kowal, C. T., Hartwick, F. D. A., \& van den Bergh, S. 1977, AJ, 82, 947

Stetson, P. B., Davis, L. E., \& Crabtree, D. R. 1990, in ASP Conf. Ser. 8, 29

Williams, B. F., \& Hodge, P. W. 2001, ApJ, 559, 851 